SOI: $1.1 /$ TAS DOI: $10.15863 /$ TAS

International Scientific Journal Theoretical \& Applied Science

p-ISSN: 2308-4944 (print) e-ISSN: 2409-0085 (online)

Year: 2017 Issue: 11 Volume: 55

Published: $21.11 .2017 \quad \underline{\text { http://T-Science.org }}$

SECTION 2. Applied mathematics.

Mathematical modeling.
Normahmad Ravshanov

Doctor of Sciences, Head of Laboratory, Tashkent University of information Technologies,

Tashkent, Uzbekistan

Utkir Saidov

Staff researcher

Tashkent University of information Technologies,

Tashkent, Uzbekistan

Uguljon Orifjonova assistent professor

Tashkent state agrarian university

Tashkent, Uzbekistan

\title{
MATHEMATICAL MODEL AND NUMERICAL ALGORITHM FOR SOLVING THE PROBLEM OF ION EXCHANGE FILTERING OF LIQUIDS
}

\begin{abstract}
A detailed analytical review of scientific papers related to the process of modeling the nonstationary technological process of filtering ionic solutions from fine particles and ionic compounds is given in the paper. To study the technological process of filtering ionic solutions, depending on their physico-chemical properties and operating modes of the filtering unit, a mathematical model, a numerical algorithm and software for computer computational experiments were developed. The proposed mathematical software makes it possible to determine the operability of the filtering equipment by means of which separation of solid particles from liquid or gas, gel particles, ions and other accompanying elements is carried out. Also, the article presents the results of computer experiments on computers in the form of graphs and the conclusions associated with them.

Key words: mathematical model, numerical method, computational experiment, ion-exchange filtration, gelparticles, suspension.

Language: Russian

Citation: Ravshanov N, Saidov U, Orifjonova U (2017) MATHEMATICAL MODEL AND NUMERICAL ALGORITHM FOR SOLVING THE PROBLEM OF ION EXCHANGE FILTERING OF LIQUIDS. ISJ Theoretical \& Applied Science, 11 (55): 144-157.

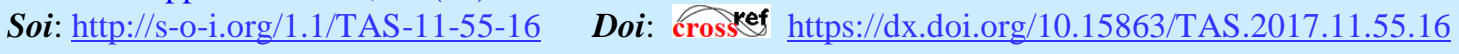

\section{МАТЕМАТИЧЕСКАЯ МОДЕЛЬ И ЧИСЛЕННЫЙ АЛГОРИТМ ДЛЯ РЕШЕНИЯ ЗАДАЧИ ИОНООБМЕННОГО ФИЛЬТРОВАНИЯ ЖИДКОСТЕЙ}

Аннотация: В работе приведен подробный аналитический обзор научных работ, связанных с прочессом моделирования нестационарного технологического прочесса фильтрования ионных растворов от мелкодисперсных частиц и ионных соединений. С иелью исследования технологического процесса фильтрования ионных растворов в зависимости от их физико-химических свойств и режимов работьл фильтрующего агрегата были разработаны математическая модель, численный алгоритм и программное обеспечение для проведения вычислительных экспериментов на ЭВМ. Предлагаемое математическое обеспечение дает возможность определять работоспособность фильтрующего оборудования с помощьью которого осуществляется отделение частии твердой фазы от жидкости или газа, гель-частиц, ионов и других сопутствующих элементов. Также, в статье приведены результаты проведенных вычислительных экспериментов на ЭВМ в виде графиков и выводы, связанные с ними.

Ключевые слова: математическая модель, численный метод, вычислительный эксперимент, ионообменная фильтрация, гель-частищь, суспензия.

\section{Введение}

Технологический процесс (ТП) фильтрования и разделения ионизированных суспензий широко распространен на заводах химической и родственных ей отраслях промышленности. А поэтому, правильная и рациональная организация управления вышеуказанным процессом позволяет существенно уменьшить эксплуатационные 


\begin{tabular}{|c|c|c|c|c|c|c|}
\hline Impact Factor: & $\begin{array}{l}\text { ISRA (India) } \\
\text { ISI (Dubai, UAE } \\
\text { GIF (Australia) } \\
\text { JIF }\end{array}$ & $\begin{array}{l}=1.344 \\
=0.829 \\
=0.564 \\
=1.500\end{array}$ & $\begin{array}{l}\text { SIS (USA) } \\
\text { PИНЦ (Russia) } \\
\text { ESJI (KZ) } \\
\text { SJIF (Morocco) }\end{array}$ & $\begin{array}{l}=0.912 \\
=0.207 \\
=3.860 \\
=\mathbf{2 . 0 3 1}\end{array}$ & $\begin{array}{l}\text { ICV (Poland) } \\
\text { PIF (India) } \\
\text { IBI (India) }\end{array}$ & $\begin{array}{l}=6.630 \\
=1.940 \\
=4.260\end{array}$ \\
\hline
\end{tabular}

расходы и улучшить качество конечного продукта.

Процесс фильтрования вязких жидкостей от примесей и технологических отходов - один из основных этапов в производстве масложировых, фармацевтических, машиностроительных, пищевых и др. продуктов. Очистка химических (прядильных), растворов жидкого топлива, питьевой воды, фармацевтических препаратов и соков и т.д. от примесей, как правило, обеспечивается применением ионных фильтров и методами фильтрования их с помощью многослойных фильтров. При фильтровании жидких растворов и суспензий используются различные по физико-механическим свойствам фильтры и фильтровальные перегородки. Работоспособность фильтрующего оборудования во многом определяется фильтрующими перегородками, с помощью которых осуществляется отделение частиц твердой фазы от жидкости или газа, гель-частиц, ионов и других сопутствующих элементов.

Технологический процесс фильтрования (ТПФ) трудноразделяемых жидкостей недостаточно изучен с теоретической и экспериментальной точек зрения. Проблема состоит в исследовании, прогнозировании и управлении процессом на основе его математического обеспечения.

Проведенные экспериментальные исследования показали, что на фильтрование жидких растворов воздействует множество параметров с различными удельными значениями. В связи с этим необходимо всесторонне исследовать данный процесс с помощью математической модели объекта и проведения вычислительных экспериментов (ВЭ) на ЭВМ с целью выявления условий для наиболее полного фильтрования жидкостей от примесей и тяжелых ионов.

По проблеме математического моделирования процесса фильтрования смесей в мире получены значительные теоретические и прикладные результаты.

В частности, в работе [1] предложена математическая модель (ММ) ТП адсорбции цефалоспорина в неподвижном слое колонки с применением оптимизации роя частиц. Для оценки параметра адсорбционного процесса решена задача вида:

$$
\frac{\partial C}{\partial t}=D_{L} \cdot \frac{\partial^{2} C}{\partial z^{2}}-v_{z} \cdot \frac{\partial C}{\partial z}-\left(\frac{1-\varepsilon}{\varepsilon}\right) \cdot \frac{3 \cdot k_{f}}{R_{p}}\left(C-C_{r=R_{p}}\right)
$$

при начальных и краевых условиях:

$$
\begin{gathered}
t=0 \rightarrow C(z, 0)=0 ; \\
z=0 \rightarrow C=C_{0}+\frac{D_{F} \cdot \varepsilon}{v_{z}} \cdot \frac{\partial C}{\partial z} ; \\
z=H \rightarrow \frac{\partial C}{\partial z}=0 .
\end{gathered}
$$

В математической постановке задачи $D_{L}$ коэффициент дисперсии; $k_{f}$ - коэффициент перемещения массы жидкого слоя; $v_{z}$ - скорость жидкости; $R_{p}$ - радиус частиц смолы; $\varepsilon$ пористость. Для численного интегрирования задачи авторами использован конечно-разностный метод Кранк-Николсона.

Результаты исследования сложных механизмов осаждения частиц в свече-фильтре приводятся в работе [2]. С целью изучения процесса фильтрования и разработки адекватного математического обеспечения объекта исследования проведены лабораторные эксперименты с подходящей частицей масляной суспензии в экспериментальном фильтре.

В работе [3] предложена феноменологическая модель глубоководной инфильтрации. Предложенная авторами ММ комбинируется с уравнением адъективной дисперсии и нелинейным уравнением кинетики рассматриваемого процесса. Поставленная задача решается численно с использованием явной конечно-разностной схемы. Полученные результаты сопоставлены с натурными экспериментами на установках «ЕРА», выполненными израильской водной компанией «Mekorot».

Авторами [4] разработана обобщённая ММ процесса адсорбции раствора сверхкритического углекислого газа на активизированном углероде. Адсорбционные кривые получены на лабораторной установке (адсорбент 10 мл), что позволило усовершенствовать математический аппарат объекта исследования. ММ была разработана с помощью уравнения сохранения баланса массы, то есть:

$$
\begin{gathered}
\varepsilon \frac{\partial c}{\partial t}=-u_{z} \frac{\partial c}{\partial z}+\varepsilon D_{a z} \frac{\partial^{2} c}{\partial z^{2}}-k_{f} a(1-\varepsilon)\left(c-\left.c_{i}\right|_{r=R_{p}}\right) \\
\beta \frac{\partial c_{i}}{\partial t}=D_{e} \frac{1}{r^{2}} \frac{\partial}{\partial r}\left(r^{2} \frac{\partial c_{i}}{\partial r}\right)-\rho_{s} \frac{\partial c_{s}}{\partial t} \\
\frac{\partial c_{s}}{\partial t}=k_{a} c_{i}-k_{d} c_{s}
\end{gathered}
$$

с начальными и краевыми условиями: 


$$
\begin{gathered}
c=0, c_{i}=0, \quad c_{s}=0 \text { при } \mathrm{t}=0, \\
c=c_{0}+\frac{\varepsilon D_{a z}}{u_{z}} \frac{\partial c}{\partial z} \quad \text { при } \quad z=0, \\
\frac{\partial c}{\partial z}=0 \quad \text { при } z=L, \\
\frac{\partial c_{i}}{\partial r}=0 \quad \text { при } \quad r=0, \\
D_{e}\left(\frac{\partial c}{\partial r}\right)_{r=R p}=k_{f}\left(c-\left.c_{i}\right|_{r=R_{p}}\right) \text { при } r=R_{p} .
\end{gathered}
$$

Здесь $a$ - определенная поверхность твердой фазы; $c_{i}$ - концентрация в порах фильтра (мол/м ${ }^{3}$ ); $c$ - концентрация раствора в жидкой фазе $\left(\right.$ моль $\left./ \mathrm{M}^{3}\right) ; c_{0}$ - начальная концентрация раствора в жидкой фазе; $c_{s}$ - концентрация раствора в твердой фазе (моль/кг); $R_{p}$ - радиус сферических частиц; $D_{a z}$ - осевой коэффициент дисперсии $\left(\mathrm{M}^{2} / \mathrm{c}\right) ; D_{e}$ - эффективный коэффициент диффузии $\left(\mathrm{M}^{2} / \mathrm{c}\right) ; \beta$ - пористость частиц; $k_{f}$ - коэффициент переноса массы в жидкой среде $(\mathrm{M} / \mathrm{c}) ; \rho_{s}$ плотность частиц $\left(\kappa г / \mathrm{M}^{3}\right) ; \varepsilon-$ коэффициент пористости.

Поставленная задача была решена с использованием метода линеаризации нелинейных членов уравнений.

В работе [5] модель баланса масса была сформирована для переноса частиц суспензий в пористых средах. Уравнения для частиц и распределения размера пор были выведены из стохастического уравнения «Master». Модель учитывает уменьшение потока частиц за счет ограничений для крупных частиц для перемещения через небольшие поры. Аналитическое решение для малоконцетрированных частиц получено для основных частиц и распределения размера пор.
Авторами
[6]
была
разработана

интегрированная ММ процесса ионообменного фильтра для очистки воды, позволяющая, оценить эффективность регенерации смолы для оценки и совершенствования ионообменной технологии. Адекватность модели была подтверждена экспериментальными данными. Воздействие безразмерных групп (т.е. число Пекле, диффузионный модуль упругости и число Байот) на ионном кривом обмене прорыв были проанализированы с использованием этой модели. Кроме того, интегральная модель была использована, чтобы оптимизировать частоту регенерации и улучшить общую производительность ионного обмена. Авторами утверждается, что интегральная модель может быть полезным инструментом для дальнейших исследований в области ионообменной технологии.

В исследовании [7] рассмотрен процесс фильтрования суспензий при использовании Amberlite IRC 748 смолы для удалении ионов кальция из высоко концентрированного раствора хромата калия в колонке с неподвижным слоем. Разработаны четыре различных кинетических модели для процесса фильтрования раствора с учетом толщины слоя и срока службы фильтровальной перегородки. В работе использованы также модели Томаса и ЮнНельсона, а результаты численных расчетов сопоставлены с экспериментальным данными, чем доказана их адекватность.

Автором [8] разработана компьютерная модель и численный алгоритм для исследования процесса многократного ионообменного фильтрования суспензий через пористую среду и проведены вычислительные эксперименты на ЭВМ при заданиях различных значений параметров ТП. На основе проведенного комплексного исследования определены время работы ионитного фильтра в зависимости от физико-химического состава подаваемой к колонке фильтра жидкости.

Приближенно-аналитическое решение задачи ТП фильтрования растворов от нежелательных ионов рассмотрено в работе [9]. Получено аналитическое решение задачи в явном виде. На основе полученного решения определен коэффициент бародиффузии, который играет существенную роль в технологии фильтрования ионных растворов.

Анализ полученных результатов исследований в выше указанных работах показал, что не до сих пор изучена степень кольматации гель-частиц в порах фильтровальной перегородки агрегата на различных глубинах при ионообменной фильтрации ионных растворов через пористую среду, а также при переменной пористости фильтра.

\section{Постановка задачи}

При выводе математической модели процесса ионообменного фильтрования жидкостей через пористую среду предполагается, что в процессе фильтрования гель-частицы оседают в порах равномерно по всей толщине фильтровальной перегородки. Однако, как показывают теоретические и экспериментальные данные, степень кольматации гель-частиц от поверхности фильтра на различных это глубинах неодинаковая. Поскольку ионообменный процесс происходит по всей толщине ионита, то решение задач с учетом степени кольматация на различных глубинах ионообменного фильтра представляет собой особый интерес. 


\begin{tabular}{l|lr|ll|ll} 
& ISRA (India) & $=\mathbf{1 . 3 4 4}$ & SIS (USA) & $=\mathbf{0 . 9 1 2}$ & ICV (Poland) & $\mathbf{6 . 6 3 0}$ \\
Impact Factor: & ISI (Dubai, UAE) $=\mathbf{0 . 8 2 9}$ & PUHI (Russia) $=\mathbf{0 . 2 0 7}$ & PIF (India) & $=\mathbf{1 . 9 4 0}$ \\
& GIF (Australia) & $=\mathbf{0 . 5 6 4}$ & ESJI (KZ) & $=\mathbf{3 . 8 6 0}$ & IBI (India) & $\mathbf{4 . 2 6 0}$ \\
& JIF & $\mathbf{1 . 5 0 0}$ & SJIF (Morocco) & $\mathbf{2 . 0 3 1}$ & & \\
\hline
\end{tabular}

Для вывода ММ процесса фильтрования жидкости через пористую среду воспользуемся уравнениями двухфазных сред.

Уравнения движения для жидкой и уравнения неразрывности для твердой фазы записываются в виде [10]:

$$
\begin{gathered}
\left(\frac{\partial}{\partial t}+\frac{\partial \vartheta_{1}}{\partial x}\right) \theta \rho \vartheta_{1}=\frac{\partial P}{\partial x}+\mu \frac{\partial^{2} \vartheta_{1}}{\partial x^{2}}-r_{1}, \\
\frac{\partial \theta_{2}}{\partial t}+\frac{\partial \theta_{2} \vartheta_{2}}{\partial x}=0 .
\end{gathered}
$$

Здесь $\vartheta_{1}$ и $\vartheta_{2}$ - компоненты скорости жидкой и твердой фаз; $\rho$ и $\mu$ - плотность и вязкость суспензий; $P$ - давление; $r_{1}$ - компонента внутренних среднестатических сил взаимодействия компонент; $\theta_{1}$ и $\theta_{2}$ - локальные концентрации жидкой и твердой фазы.
К уравнениям (1) и (2) добавляется уравнение связи

$$
\theta_{1}+\theta_{2}=1
$$

Из уравнения неразрывности жидкой фазы следует, что

$$
\begin{aligned}
\theta_{1}^{(1)} \vartheta_{1}^{(1)}= & \theta_{1}^{(2)} \vartheta_{1}^{(2)}=W\left[1-\theta_{2}^{(3)}(t)\right] ; \\
& \left(1-\theta_{2}^{(1)}\right) \vartheta_{1}^{(1)}=\left(1-\theta_{2}^{(3)}\right) \theta_{1}^{(3)} .
\end{aligned}
$$

В случае движения «нейтральных» частиц суспензии имеем:

$$
\left.\begin{array}{l}
\vartheta_{2}^{(1)}=\vartheta_{2}^{(1)} \\
\vartheta_{2}^{(3)}=\vartheta_{1}^{(3)}=W
\end{array}\right\}
$$

Здесь $\vartheta_{2}^{(\kappa)}\left(\vartheta_{2}^{(\kappa)}\right)$ и $\theta_{1}^{(\kappa)}, \theta_{2}^{(\kappa)}$ - скорость и концентрация жидкой и твердой фаз в области $\mathcal{D}_{\kappa}$ (рис.1); $W$ - скорость фильтрования.

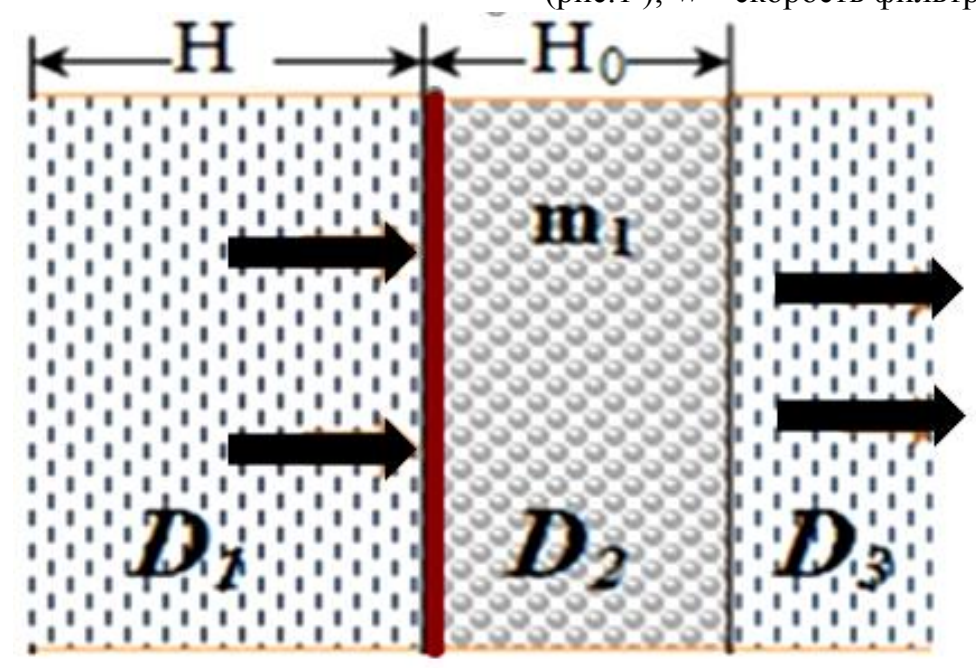

Рисунок 1 - Скорость и концентрация жидкой и твердой фаз.

Уравнения (1), (2) с учетом соотношений (3)(4) представляются в виде

$$
\begin{gathered}
\frac{\partial W\left(1-\theta^{(3)}\right)}{\partial t}+W \frac{\partial W\left(1-\theta^{(3)}\right)}{\partial x}= \\
=\frac{\left(1-\theta^{(3)}\right)}{\rho} \frac{\partial P}{\partial x}+\frac{\mu}{\rho} \frac{\partial^{2} W\left(1-\theta^{(3)}\right)}{\partial x^{2}}-r_{1}, \\
\frac{\partial \theta}{\partial t}+\frac{\partial \theta W}{\partial x}=0 .
\end{gathered}
$$

Здесь $\quad r_{1}=\frac{\mu \mathrm{H}_{0} W}{k_{0}\left[1-\xi^{*}(x, t)\right]}, \quad \mathrm{H}_{0}-$ толщина фильтра; $k_{0}{ }^{-}$коэффициент проницаемости фильтра до начала его работы; $\xi^{*}=\frac{\mathrm{k}(x, t)}{\mathrm{k}_{0}}-$ коэффициент проницаемости фильтра во время его работы.
В дальнейшем, для удобства записи в уравнениях нижний индекс концентрации жидкой и твердой фаз будет опущен.

Пусть $\theta^{(3)}=\theta(t)^{(3)}$. Тогда из первого уравнения (5) получим:

$$
\begin{gathered}
\frac{\partial W}{\partial t}+W \frac{\partial W}{\partial x}-\frac{\partial \theta^{(3)}}{\partial t}= \\
=-\frac{1}{\rho} \frac{\partial P}{\partial x}+\frac{\mu}{\rho} \frac{\partial^{2} W}{\partial x^{2}}-\frac{r_{1}}{\left(1-\theta^{(3)}\right)} .
\end{gathered}
$$

Коэффициент $\kappa(x, t)$ выражается формулой

$$
\kappa(x, t)=\kappa_{0}(1-\sqrt{\xi})^{3} .
$$

Таким образом, уравнения (6 )-(7) образуют замкнутую систему для определения всех неизвестных.

Задачи (6)-(7) будем решать с краевыми условиями: 


\begin{tabular}{|c|c|c|c|c|c|c|}
\hline Impact Factor: & $\begin{array}{l}\text { ISRA (India) } \\
\text { ISI (Dubai, UAE } \\
\text { GIF (Australia) } \\
\text { JIF }\end{array}$ & $\begin{array}{l}=1.344 \\
=0.829 \\
=0.564 \\
=1.500\end{array}$ & $\begin{array}{l}\text { SIS (USA) } \\
\text { PИНЦ (Russia) } \\
\text { ESJI (KZ) } \\
\text { SJIF (Morocco) }\end{array}$ & $\begin{array}{l}=0.912 \\
=0.207 \\
=3.860 \\
=\mathbf{2 . 0 3 1}\end{array}$ & $\begin{array}{l}\text { ICV (Poland) } \\
\text { PIF (India) } \\
\text { IBI (India) }\end{array}$ & $\begin{array}{l}=6.630 \\
=1.940 \\
=4.260\end{array}$ \\
\hline
\end{tabular}

$$
\begin{aligned}
& W=W_{0}, \theta^{(3)}=0, \quad \theta=\theta^{(1)} e^{-\lambda m_{1} \frac{1-m_{0}}{W_{0}} x}, \\
& \xi=0, \quad(t=0) \\
& W=W_{0}, \theta=\theta^{(1)}, \quad(x=0) ; \\
& \frac{\partial W}{\partial x}=\varphi_{1},(x=1) .
\end{aligned}
$$

Для вывода ММ ионообменного процесса фильтрования добавляем уравнение ионообменной сорбции, которое имеет следующий вид [11-12]:

$$
\begin{gathered}
\frac{\partial n_{i} m}{\partial t}+\frac{\partial n_{i} W}{\partial t}+\frac{\partial \mathcal{N}_{1}}{\partial t}=\mathcal{D}_{\mathcal{L}} \frac{\partial^{2} n_{i}}{\partial x^{2}}, \\
\frac{\partial \mathcal{N}_{1}}{\partial t}=\beta\left(n_{i}-n_{i}^{\prime}\right), \quad \mathcal{N}_{1}=\frac{\mathrm{n}_{i}^{\prime}}{a+b}, i=1,2 \\
n_{1}+n_{2}=n_{0}, \mathcal{N}_{1}+\mathcal{N}_{2}=\mathcal{N}_{0},
\end{gathered}
$$

где $n_{1} u n_{2}{ }^{-}$неравновесные концентрации обменивающихся ионов в растворе в единице длины сорбционной колонны; $\mathcal{N}_{1} u \mathcal{N}_{2}$ неравновесные концентрации обменивающихся ионов в сорбенте; $n_{1} u n_{2}$ - концентрации ионов в растворе, соответствующие равновесию с концентрациями $\mathcal{N}_{1} u \mathcal{N}_{2} ; \quad \beta$ - эффективная константа обменивающихся ионов; $\mathcal{D}_{\mathcal{L}}$ коэффициент продольной диффузии; $a$ и $b$ постоянные изотермы; $n_{0}$ - исходная концентрация в растворе вводимого в колонну иона; $\mathcal{N}_{0}$ - обменная емкость поглощения сорбента.

Для определения $\xi(x, t)$ скорости осаждения гель-частиц по глубине фильтровальной колонки фильтра воспользуемся уравнениями баланса и кинетики для нестационарного случая:

$$
\begin{gathered}
\frac{\partial \theta m}{\partial t}+\frac{\partial \theta W}{\partial x}+\frac{\partial \alpha m}{\partial t}+\left(1-m_{0}\right) \frac{\partial \xi m}{\partial t}=0, \\
\frac{\partial \xi}{\partial t}=\lambda(\theta-\gamma \xi) .
\end{gathered}
$$

Здесь $\lambda$ - кинетический коэффициент; $\gamma$ - в отличие от коэффициента Генри для поглощения газов, зависит от величины и состояния поверхности поровых каналов и в соответствии с опытными данными может быть представлен как

$$
\gamma=\frac{a_{0}}{b_{0}}
$$

Здесь $a_{0}$ изменяется от 0,0005 до $0,0036, b_{0}$ - от 0,7 до 0,9 , а $m_{0}$ - пористость осевшей массы.

Из уравнения баланса и кинетики процесса можно вычислить $\theta^{3}(t)$

$$
\frac{\partial \theta^{3}(t)}{\partial t}=\frac{\left(m_{1}-m_{0}\right)\left(1-m_{0}+\theta^{3}(t)\right)}{m_{1}+\left(m_{1}-m_{0}\right)} \frac{\partial \xi}{\partial t} .
$$

Здесь учтено, что $m=m_{1}+\left(m_{1}-m_{0}\right) \xi$, и при переменной пористости, уравнения неразрывности записывается в виде

$$
\frac{\partial \theta m}{\partial t}+\frac{\partial \theta W}{\partial x}=0
$$

где $m_{1}$ - пористость фильтра до его работы.

Градиент давления, входящий в уравнение (7) определяется из закона Дарси

$$
W=-\frac{\kappa(x, t)}{\mu} \frac{\partial \mathrm{P}}{\partial x}
$$

Теперь определим $\theta^{(3)}(t)$ и $\xi^{*}(x, t)$. Среда и суспензия, таковы что в процессе фильтрования часть твердого вещества взвести задерживается средой, часть ранее осевших частиц срывается и попадает в фильтрационный поток, а часть переносится фильтрационным потоком в области ( $\mathcal{D}^{3}$ ). Обозначим через $\alpha$ насыщенность порового пространства взвешенным частицами, через $\xi$ осевшие в рыхлом теле частицы. Тогда концентрация взвешенного твердого вещества в движущейся смеси будет

$$
\theta=\frac{\alpha}{1-\xi} \text {. }
$$

Так как каждая частица взвеси попеременного может находиться в покое и во взвешенном состоянии, то чтобы выразит $\alpha$ через $\theta^{(3)}$ и $\xi$ в общем случае, нужно применить вероятное осреднение.

Для численного интегрирования задачи, упростим ее. Пусть на достаточной глубине от поверхности фильтра частицы под действием силы потока и давления не срываются, взвеси в порах фильтра равно количеству срывающихся частиц. Тогда в уравнении (12) $\alpha$ можно заменить на $\theta^{(3)}$, т.е.

$$
\theta=\frac{\theta^{(3)}(t)}{1-\xi(x, t)}
$$

В уравнениях (9) и (10) индекс «i» относится к иону, насыщающему сорбент в исходном состоянии, а индекс «2» - к иону, входящему в колонну и вытесняющему первый ион.

Краевые условия задачи (9)-(10) следующие:

$n_{1}=0, n_{2}=n_{0}, \mathcal{N}_{1}=\mathcal{N}_{0}, \mathcal{N}_{2}=0,(t=0) ;$

$n_{1}=0, n_{2}=n_{0},(x=0)$;

$n_{1}=n_{0}, n_{2}=0,(x=1)$.

В силу уравнения эквивалентности обмена (11) достаточно найти решение для одного из 


\begin{tabular}{|c|c|c|c|c|c|c|}
\hline Impact Factor: & $\begin{array}{l}\text { ISRA (India) } \\
\text { ISI (Dubai, UAE } \\
\text { GIF (Australia) } \\
\text { JIF }\end{array}$ & $\begin{array}{l}=1.344 \\
=0.829 \\
=0.564 \\
=1.500\end{array}$ & $\begin{array}{l}\text { SIS (USA) } \\
\text { PИНЦ (Russia) } \\
\text { ESJI (KZ) } \\
\text { SJIF (Morocco) }\end{array}$ & $\begin{array}{l}=\mathbf{0 . 9 1 2} \\
=\mathbf{0 . 2 0 7} \\
=\mathbf{3 . 8 6 0} \\
=\mathbf{2 . 0 3 1}\end{array}$ & $\begin{array}{l}\text { ICV (Poland) } \\
\text { PIF (India) } \\
\text { IBI (India) }\end{array}$ & $\begin{array}{l}=6.630 \\
=1.940 \\
=4.260\end{array}$ \\
\hline
\end{tabular}

обменивающихся ионов. Для удобства решения задачи введем безразмерные переменные

$$
\begin{gathered}
\bar{W}=\frac{W}{W_{0}}, q=\frac{\mathrm{H}_{0}}{\mu W_{0}} P, \bar{n}=\frac{n}{\mathcal{N}_{0}} \bar{x}=\frac{x}{\mathrm{H}_{0}}, \quad t=\alpha_{\tau} \tau \\
\alpha_{\tau}=\frac{P F}{\mu}, \bar{\theta}^{(3)}=\frac{\theta^{(3)}}{\theta^{(1)}}, \bar{\xi}=\frac{\xi}{\theta^{(1)}}, \bar{\theta}=\frac{\theta}{\theta^{(1)}},
\end{gathered}
$$

и получим [12-14]:

$$
\begin{aligned}
& \frac{\partial W}{\partial q}+R e W \frac{\partial W}{\partial x}-\frac{W}{\left(1-\theta^{(3)}\right)}= \\
& =\frac{F}{\mathrm{H}_{0}{ }^{2}}\left(-\frac{\partial q}{\partial x}+\frac{\partial^{2} W}{\partial x^{2}}-\frac{\mathrm{H}_{0} F}{\mathrm{H} K_{0}\left(1-\theta^{(3)}\right)} \frac{W}{(1-\xi)^{2}}\right) \text {; } \\
& \frac{\partial \theta}{\partial t}+R e \frac{\partial \theta W}{\partial x}=0 ; \\
& \frac{\partial \theta^{(3)}}{\partial \tau}=\frac{K_{1}\left(K_{2}+\theta^{(3)}\right)}{K_{3}+K_{4} \xi} \frac{\partial \xi}{\partial \tau} ; \\
& \frac{\partial \xi}{\partial \tau}=\lambda_{1}(\theta-\gamma \xi) \\
& W=\frac{K}{{\mathrm{H}_{0}}^{2}} \frac{\partial q}{\partial x} ; \\
& \frac{\partial n m}{\partial q}+R e \frac{\partial n W}{\partial x}+\frac{\partial N}{\partial q}=a_{0} \frac{\partial^{2} n}{\partial x^{2}} ; \\
& \frac{\partial N}{\partial q}=\beta \alpha_{q}\left(n-\frac{\alpha_{1} N}{\alpha_{2}-b N}\right) \\
& W=1, \theta^{(3)}=0, \theta=e^{-b_{0} x}, \xi=0,(\tau=0) \text {; } \\
& W=1, \theta=1,(x=0) \text {; } \\
& \frac{\partial W}{\partial q}=\varphi,(x=1) \text {; } \\
& \left.\begin{array}{l}
n(x, 0)=0, N(x, 0)=1 ; \\
n(x, q)=0, n(1, q)=\frac{n_{0}}{N_{0}}=n^{0} .
\end{array}\right\}
\end{aligned}
$$

Здесь $R e=\frac{\rho F W_{0}}{\mu \mathrm{H}_{0}}-$ число Рейнольдса; $\kappa_{1}=m_{1}-m_{0}, \quad \kappa_{2}=\frac{1-m_{0}}{\theta^{(1)}}, \quad \kappa_{3}=\frac{m_{1}}{\theta^{(1)}}, \quad \kappa_{4}=-\kappa_{1}$, $a_{1}=\frac{a}{N_{0}}, a_{2}=\frac{1}{N_{0}}, \varphi=\varphi_{1} \frac{\mathrm{H}_{0}}{W_{0}}, b_{0}=\lambda_{1} m_{1} \mathrm{H}_{0} \frac{1-m_{0}}{W_{0}}$ , $a_{0}=\frac{\mathcal{D}_{L} F \rho}{\mu \mathrm{H}_{0}}, F-$ площадь фильтра.

Здесь и в дальнейшем для удобства записи черточки над безразмерным и переменными будут опущены.

\section{Метод решения}

Систему (16)-(23) интегрировать аналитически не представляется возможным. Поэтому применим численный метод ее интегрирования.

Для уравнения (16) с соответствующим краевым условиями (22) применим векторную схему Самарского-Фрязинова [15-16]:

$$
\begin{aligned}
& \frac{\partial u}{\partial \tau}+\operatorname{Re} u \frac{\partial v}{\partial x}-\frac{u}{1-\theta_{u}^{(3)}} \frac{\partial \theta_{u}^{(3)}}{\partial \tau}= \\
& =\frac{F}{\mathrm{H}_{0}^{2}}\left(-\frac{\partial q_{u}}{\partial x}+\frac{\partial^{2} u}{\partial x^{2}}\right)- \\
& -\frac{\mathrm{H}_{0} F}{\mathrm{H} K_{0}\left(1-\theta_{u}^{(3)}\right)} \frac{u}{\left(1-\xi_{u}\right)^{2}}, \\
& \frac{\partial v}{\partial \tau}+\operatorname{Rev} \frac{\partial u}{\partial x}-\frac{v}{1-\theta_{v}^{(3)}} \frac{\partial \theta_{v}^{(3)}}{\partial \tau}= \\
& =\frac{F}{\mathrm{H}_{0}^{2}}\left(-\frac{\partial \theta_{v}}{\partial x}+\frac{\partial^{2} v}{\partial x^{2}}\right)- \\
& -\frac{\mathrm{H}_{0} F}{\mathrm{H} K_{0}\left(1-\theta_{v}^{(3)}\right)} \frac{v}{\left(1-\xi_{v}\right)^{2}}, \\
& \mathrm{u}(x, 0)=v(x, 0)=1, u(0, \tau)=v(0, \tau)=1 ; \\
& \left.\frac{\partial u}{\partial x}\right|_{x=1}=\left.\frac{\partial v}{\partial x}\right|_{x=1}=\varphi
\end{aligned}
$$

Для получения второго порядка аппроксимации по пространственной переменной, уравнение (17) записываем в виде:

$$
\left.\begin{array}{l}
\frac{\partial y}{\partial \tau}+\lambda \frac{\partial z}{\partial x}+\operatorname{Re} y \frac{\partial u}{\partial x}=0 \\
\frac{\partial z}{\partial \tau}+\lambda \frac{\partial y}{\partial x}+\operatorname{Re} z \frac{\partial v}{\partial x}=0
\end{array}\right\}
$$

В первом уравнении (27) имеет положительный знак, а во втором отрицательный. Поэтому обозначим их соответственно через $\lambda^{+}$и $\lambda^{-}$.

Краевые условия этой системы будут следующие

$$
\left.\begin{array}{l}
y(x, 0)=z(x, 0)=e^{-b_{0} x} \\
y(0, \tau)=z(0, \tau)=1
\end{array}\right\}
$$

Уравнения (18) и (19) представляются в виде:

$$
\left.\begin{array}{l}
\frac{\partial \theta_{u}^{(3)}}{\partial \tau}=\frac{K_{1}\left(K_{2}+\theta_{u}^{(3)}\right)}{K_{3}+K_{4} \varepsilon_{u}} \frac{\partial \xi_{u}}{\partial \tau}, \\
\frac{\partial \theta_{v}^{(3)}}{\partial \tau}=\frac{K_{1}\left(K_{2}+\theta_{v}^{(3)}\right)}{K_{3}+K_{4} \varepsilon_{v}} \frac{\partial \xi_{v}}{\partial \tau}, \\
\theta_{u}^{(3)}=\theta_{v}^{(3)}=0
\end{array}\right\}
$$




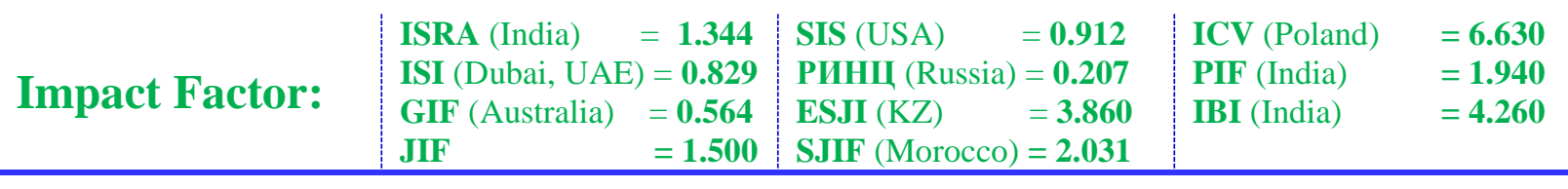

$$
\begin{aligned}
& \left.\begin{array}{l}
\frac{\partial \xi_{u}}{\partial \tau}=\lambda_{1}\left(y-\gamma \xi_{u}\right) ; \\
\frac{\partial \xi_{v}}{\partial \tau}=\lambda_{1}\left(z-\gamma \xi_{v}\right) ; \\
\xi_{u}=\xi_{v}=0
\end{array}\right\} \\
& \frac{\partial m_{u} n^{(1)}}{\partial \tau}+\operatorname{Re} \frac{\partial u n^{(2)}}{\partial x}+\frac{\partial N^{(1)}}{\partial \tau}=a_{0} \frac{\partial^{2} n^{(1)}}{\partial x^{2}} ; \\
& \frac{\partial N^{(1)}}{\partial \tau}=\beta \alpha_{\tau}\left(n^{(1)}-\frac{a_{1} N^{(1)}}{a_{2}-b N^{(2)}}\right) ; \\
& \frac{\partial m_{v} n^{(2)}}{\partial \tau}+\operatorname{Re} \frac{\partial v n^{(2)}}{\partial x}+\frac{\partial N^{(1)}}{\partial \tau}=a_{0} \frac{\partial^{2} n^{(2)}}{\partial x^{2}} ; \\
& \frac{\partial N^{(2)}}{\partial \tau}=\beta \alpha_{\tau}\left(n^{(2)}-\frac{a_{1} N^{(2)}}{a_{2}-b N^{(2)}}\right) ; \\
& n^{(1)}(x, 0)=n^{(2)}(x, 0)=0 \text {; } \\
& N^{(1)}(x, 0)=N^{(2)}(x, 0)=1 \text {; } \\
& n^{(1)}(0, \tau)=n^{(2)}(0, \tau)=0 \text {; } \\
& n^{(1)}(1, \tau)=n^{(2)}(1, \tau)=n^{0} \text {. }
\end{aligned}
$$

Аппроксимируем уравнение (24) и краевые условия (25) разностной схемой, при этом $\frac{\partial u}{\partial x}$ двухточечной схемой вперед, а $\frac{\partial v}{\partial x}$ - той же назад и получим [13-14]:

$$
\begin{array}{r}
u_{i}=A_{i+1} u_{i+1}+B_{i+1} v_{i+1}+C_{i+1} ; \\
v_{i}=A_{i+1}^{\prime} v_{i+1}+B_{i+1}^{\prime} u_{i+1}+C_{i+1}^{\prime} ; \\
A_{1}=A_{1}^{\prime}=B_{1}=B_{1}^{\prime}=0, C_{1}=C_{1}^{\prime}, \\
u_{p}=\frac{\mathcal{G}_{13} \mathcal{G}_{21}-\mathcal{G}_{12} \mathcal{G}_{23}+2 h \psi\left(\mathcal{G}_{21}-\mathcal{G}_{12}\right)}{\mathcal{G}_{11} \mathcal{G}_{21}-\mathcal{G}_{12} \mathcal{G}_{23}}, \\
v_{p}=\frac{\mathcal{G}_{23} \mathcal{G}_{11}-\mathcal{G}_{13} \mathcal{G}_{22}+2 h \psi\left(\mathcal{G}_{11}-\mathcal{G}_{22}\right)}{\mathcal{G}_{11} \mathcal{G}_{21}-\mathcal{G}_{12} \mathcal{G}_{23}},
\end{array}
$$

где

$$
\begin{gathered}
\mathrm{G}_{11}=3+\left(\mathrm{A}_{p-2}-4\right) \mathrm{A}_{p-1}+B_{p-2} B_{p-1}^{\prime}, \\
\mathcal{G}_{12}=\left(\mathrm{A}_{p-2}-4\right) \mathrm{B}_{p-1}+B_{p-2} A_{p-1}^{\prime}, \\
\mathcal{G}_{13}=\left(\mathrm{A}_{p-2}-4\right) \mathrm{C}_{p-1}+B_{p-2} C_{p-1}^{\prime}+\mathrm{C}_{p-2}, \\
\mathcal{G}_{21}=3+\left(A_{p-2}^{\prime}-4\right) A_{p-1}^{\prime}+B_{p-1} B_{p-1}^{\prime}, \\
\mathcal{G}_{22}=\left(A_{p-2}^{\prime}-4\right) B_{p-1}^{\prime}+A_{p-1} B_{p-2}^{\prime}, \\
\mathcal{G}_{23}=\left(A_{p-2}^{\prime}-4\right) C_{p-1}^{\prime}+C_{p-1} B_{p-2}^{\prime}+C_{p-2}^{\prime},
\end{gathered}
$$

Аппроксимируем (27) явной схемой. Первое уравнения - разностной схемой вперед, а второе назад:

$$
\left.\begin{array}{l}
\mathcal{Y}_{i}=E_{i+1} y_{i+1}+D_{i+1} z_{i+1}+F_{i+1}, \\
\mathcal{Z}_{i}=E_{i+1}^{\prime} z_{i+1}+D_{i+1}^{\prime} y_{i+1}+F_{i+1}^{\prime} .
\end{array}\right\}
$$

Коэффициенты, входящие в уравнения (31), (32) для постоянного градиента давления определяются из рекуррентных соотношений:

$$
\begin{aligned}
& \mathrm{A}_{i+1}=\frac{1}{R_{i}}\left[a_{i}\left(b_{i}^{\prime}-c_{i}^{\prime} A_{i}^{\prime}\right)-d_{i}^{\prime}\left(c_{i} B-d_{i}+e_{i} A_{i}^{\prime}\right)\right], \\
& \mathrm{B}_{i+1}=\frac{1}{R_{i}}\left(c_{i} B_{i}-d_{i}+e_{i} A_{i}^{\prime}\right), \\
& C_{i+1}=\frac{1}{R_{i}}\left[\left(c_{i} C_{i}+e_{i} C_{i}^{\prime}+f_{i}\right)\left(b_{i}^{\prime}-c_{i}^{\prime} A_{i}^{\prime}\right)+\right. \\
& \left.+\left(c_{i} B_{i}-d_{i}+e_{i} A_{i}^{\prime}\right)\left(c_{i} C_{i}^{\prime}+f_{i}^{\prime}\right)\right] \\
& A_{i+1}^{\prime}=\frac{a_{i}}{R_{i}}\left(b_{i}-c_{i} \mathrm{~A}_{i}-e_{i} B_{i}^{\prime}\right), \\
& B_{i+1}^{\prime}=\frac{1}{R_{i}}\left[a_{i}^{\prime}\left(e_{i}^{\prime}+c_{i}^{\prime} B_{i}^{\prime}\right)-d_{i}^{\prime}\left(b_{i}-C_{i} \mathrm{~A}_{i}-e_{i} B_{i}^{\prime}\right)\right] \\
& C_{i+1}^{\prime}=\frac{1}{R_{i}}\left[\left(b_{i}-c_{i} \mathrm{~A}_{i}-e_{i} B_{i}^{\prime}\right)\left(c_{i} C_{i}^{\prime}+f_{i}^{\prime}\right)+\right. \\
& \left.+\left(e_{i}^{\prime}+c_{i}^{\prime} B_{i}^{\prime}\right)\left(c_{i} C_{i}+e_{i} C_{i}^{\prime}+f_{i}\right)\right], \\
& R_{i}=\left(b_{i}-c_{i} \mathrm{~A}_{i}-e_{i} B_{i}^{\prime}\right)\left(-c_{i}^{\prime} A_{i}^{\prime}\right)- \\
& -\left(c_{i} B_{i}-d_{i}+e_{i} A_{i}^{\prime}\right)\left(e_{i}^{\prime}+c_{i}^{\prime} B_{i}^{\prime}\right) \text {, } \\
& a_{i}=a_{i}^{\prime}=c_{i}=c_{i}^{\prime}=\frac{1}{h^{2}}, d_{i}=e_{i}=\operatorname{Re} \frac{U_{i}^{s-1}}{h}, \\
& d_{i}^{\prime}=e_{i}^{\prime}=\operatorname{Re} \frac{v_{i}^{s-1}}{h}, \\
& f_{i}=\frac{U_{1}}{\Delta q}+\frac{\partial q}{\partial x} \cdot \frac{F}{\mathrm{H}_{0}{ }^{2}}, f_{i}^{\prime}=\frac{v_{1}}{\Delta q}+\frac{F}{\mathrm{H}_{0}{ }^{2}} \cdot \frac{\partial q}{\partial x}, \\
& b_{i k}=\frac{1}{\Delta q}+\frac{2}{h^{2}}+\frac{\mu \mathrm{H}_{0} F}{\mathrm{HK}_{0}\left(1-\tilde{\theta}_{i k}^{(3)}\right)\left(1-\varepsilon_{i k}\right)^{2}}- \\
& -\frac{1}{1-\tilde{\theta}_{i k}^{(3)}} \frac{\tilde{G}_{i k}^{2}+P_{3 k} \tilde{G}_{i k}-P_{4 k} \tilde{\theta}_{i k}^{(3)}}{\Delta q}, \\
& \mathrm{D}_{i+1}=\frac{\bar{a}_{i}^{\prime}}{R_{1 i}}\left(\bar{b}_{i}^{\prime}-\bar{c}_{i} D_{i}^{\prime}\right), D_{i+1}^{\prime}=\frac{\bar{a}_{i}^{\prime}}{R_{1 i}}\left(\bar{b}_{i}^{\prime}-\bar{c}_{i}^{\prime} D_{i}^{\prime}\right), \\
& \mathrm{E}_{i+1}=\frac{\bar{a}_{i}^{\prime}}{R_{1 i}}\left(\bar{d}_{i}-\bar{c}_{i} E_{i}^{\prime}\right) E_{i+1}^{\prime}=\frac{\bar{a}_{i}^{\prime}}{R_{1 i}}\left(\bar{d}_{i}^{\prime}+\bar{c}_{i}^{\prime} E_{i}^{\prime}\right),
\end{aligned}
$$




$$
\begin{aligned}
& \mathrm{F}_{i+1}=\frac{1}{R_{1 i}}\left[\left(\bar{c}_{i} F_{i}+\bar{f}_{i}\right)\left(\bar{b}_{i}^{\prime}-\bar{c}_{i}^{\prime} D_{i}^{\prime}\right)+\right. \\
& \left.+\left(\bar{d}_{i}+\bar{c}_{i} E_{i}^{\prime}\right)\left(\bar{c}_{i}^{\prime} F_{i}^{\prime}+\bar{f}_{i}^{\prime}\right)\right], \\
& E_{i+1}^{\prime}=\frac{1}{R_{1 i}}\left[\left(\bar{c}_{i}^{\prime} \bar{F}_{i}^{\prime}+\bar{f}_{i}^{\prime}\right)\left(\bar{b}_{i}^{\prime}-\bar{c}_{i}^{\prime} D_{i}\right)+\right. \\
& \left.+\left(\bar{d}_{i}^{\prime}+\bar{c}_{i}^{\prime} E_{i}^{\prime}\right)\left(\bar{c}_{i}^{\prime} F_{i}+\bar{f}_{i}\right)\right], \\
& R_{1 i}=\left(\bar{b}_{i}^{\prime}-\bar{c}_{i} D_{i}\right)\left(\bar{b}_{i}^{\prime}-\bar{c}_{i}^{\prime} D_{i}^{\prime}\right)- \\
& -\left(\bar{d}_{i}^{\prime}+\bar{c}_{i}^{\prime} E_{i}^{\prime}\right)\left(\bar{d}_{i}+\bar{c}_{i} E_{i}\right), \\
& \bar{a}_{i 1}=\bar{c}_{i 1}=\frac{\left|\lambda_{k}\right|-\lambda_{k}}{2}, \bar{a}_{i 2}=\bar{c}_{i 2}=\frac{\left|\lambda_{k}\right|+\lambda_{k}}{2} \text {, } \\
& \bar{d}_{i k}=\bar{e}_{i k}=-\left|\lambda_{k}\right|, \bar{b}_{i k}=-\left(1-G_{k}\right), \\
& \bar{f}_{i}=-\tilde{\mathcal{Y}}_{i}, \bar{f}_{i}^{\prime}=-\tilde{\mathcal{Z}}_{i}, \\
& \lambda_{k}=\left\{\begin{array}{cc}
u_{i} \frac{\operatorname{Re} \Delta \tau}{h} & \text { при } \kappa=1 \\
v_{i} \frac{\operatorname{Re} \Delta \tau}{h} & \text { при } \kappa=2
\end{array},\right. \\
& \sigma_{k}= \begin{cases}\frac{\operatorname{Re} \Delta \tau}{h}\left(u_{i}-u_{i-1}\right) & \text { при } \kappa=1 \\
\frac{\operatorname{Re} \Delta \tau}{h}\left(v_{i+1}-v_{i}\right) & \text { при } \kappa=2\end{cases} \\
& \bar{Q}_{i k}=\left\{\begin{array}{ll}
\bar{Q}_{i} & \text { при } \kappa=1 \\
\bar{Q}_{i}^{\prime} & \text { при } \kappa=2
\end{array}, \bar{C}_{i k}=\left\{\begin{array}{ll}
\bar{C}_{i} & \text { при } \kappa=1 \\
\bar{C}_{i}^{\prime} & \text { при } \kappa=2
\end{array},\right.\right. \\
& \bar{b}_{i k}= \begin{cases}\bar{b}_{i} & \text { при } \kappa=1 \\
\bar{b}_{i}^{\prime} & \text { при } \kappa=2\end{cases} \\
& \theta_{i k}^{(3)}=\left\{\begin{array}{ll}
\theta_{y}^{(3)} & \text { при } \kappa=1 ; \\
\theta_{z}^{(3)} & \text { при } \kappa=2 ;
\end{array} \quad G_{i k}^{(3)}= \begin{cases}y_{i} & \text { при } \kappa=1 ; \\
z_{i} & \text { при } \kappa=2 .\end{cases} \right.
\end{aligned}
$$

Аппроксимируя (29) находим:

$$
\begin{gathered}
\xi_{i k}=\frac{\lambda_{1} \Delta \tau}{1-\lambda_{1} \Delta \tau} \mathrm{G}_{i k}+\frac{\bar{\xi}_{i k}}{1-\lambda_{1} \Delta \tau}, \\
\theta_{i k}^{(3)}=\frac{K_{1} K_{2}\left(\xi_{i k}-\xi_{i k}\right)}{K_{3}+K_{1} \xi_{i k}+\left(K_{2}-K_{1}\right) \xi_{i k}},
\end{gathered}
$$

где $\xi_{i k}= \begin{cases}\xi_{i u} & \text { при } \kappa=1 ; \\ \xi_{i v} & \text { при } \kappa=2 .\end{cases}$

Системы решаются методом совместной прогонки. Для вычисления значения $\mathcal{Y}_{p+1}$ и $\mathcal{Z}_{p+1}$, используем явную схему и найдем:

$$
\left.\begin{array}{l}
y_{p+1}=\frac{h_{1}-h_{2} h_{3}}{1-h_{2} h_{4}} \\
z_{p+1}=\frac{h_{3}-h_{1} h_{4}}{1-h_{2} h_{4}}
\end{array}\right\}
$$

где

$$
\begin{gathered}
h_{1}=\frac{\lambda_{0}\left[F_{p-2}^{\prime}-F_{p-1}^{\prime}\left(1-E_{p-2}^{\prime}\right)\right]-F_{p-1}\left(h_{0}-\lambda_{0} D_{p-2}^{\prime}\right)}{E_{p-1}\left(h_{0}-\lambda_{0} D_{p-1}^{\prime}\right)+\lambda_{0} D_{p-1}^{\prime}\left(1-E_{p-2}^{\prime}\right)}, \\
h_{2}=\frac{D_{p-1}\left(h_{2}-\lambda_{0} D_{p-2}^{\prime}\right)+\lambda_{0} E_{p-1}^{\prime}\left(1-E_{p-2}^{\prime}\right)}{E_{p-1}\left(h_{0}-\lambda_{0} D_{p-2}^{\prime}\right)+\lambda_{0} D_{p-1}^{\prime}\left(1-E_{p-2}^{\prime}\right)}, \\
h_{3}=\frac{\lambda_{0} F_{p-1}-h F_{p-1}^{\prime}}{h E_{p-1}^{\prime}-\lambda_{0} D_{p-1}}, h_{3}=\frac{h D_{p-1}^{\prime}+\lambda_{0}\left(1-E_{p-1}\right)}{h E_{p-1}^{\prime}-\lambda_{0} D_{p-1}}, \\
\lambda_{0}=\frac{\lambda_{1} \Delta q}{h}, h_{0}=1+\operatorname{Re} \frac{\Delta q}{h} \frac{U_{p-1}-U_{p-2}}{h} \\
h=1+\operatorname{Re} \frac{\Delta q}{h} \frac{v_{p}-v_{p-1}}{h} .
\end{gathered}
$$

Из краевых условий найдем

$$
E_{1}=E_{1}^{\prime}=D_{1}=D_{1}^{\prime}=0, F_{1}=F_{1}^{\prime}=1 .
$$

Для решения уравнения ионообменной сорбции (21) с начальными и граничными условиями (23) применим векторную схему Фрязинова и аппроксимируя полученное с точностью $O\left(h^{2}\right)$, найдем

$$
\left.\begin{array}{l}
n_{1}^{(1)}=A_{i+1} n_{i+1}^{(1)}+B_{i+1} n_{i+1}^{(2)}+C_{i+1}, \\
n_{1}^{(2)}=A_{i+1}^{\prime} n_{i+1}^{(2)}+B_{i+1}^{\prime} n_{i+1}^{(1)}+C_{i+1}^{\prime}
\end{array}\right\}
$$

в которых значения малых коэффициентов имеют вид:

$$
\begin{gathered}
a_{i}=a_{i}^{\prime}=c_{i}=c_{i}^{\prime}=\frac{1}{h^{2}}, d_{i}=e_{i}=\frac{\operatorname{Re}}{h} u_{i}, \\
d_{i}^{\prime}=e_{i}^{\prime}=\frac{\operatorname{Re}}{h} v_{i}, \\
b_{i k}=\left\{\begin{array}{c}
\frac{2}{h^{2}}+\frac{m_{y}+\alpha_{i}+\left(m_{0}-m_{1}\right)\left(P_{0} y+P_{1} y\right)}{\Delta \tau}+ \\
\frac{+\frac{u_{i}+u_{i+1}}{h} ;}{h^{2}}+\frac{m_{z}+\alpha_{i}+\left(m_{0}-m_{1}\right)\left(P_{0} z_{i}+P_{1} z\right)}{\Delta \tau}+ \\
\quad+\frac{v_{i+1}-v_{1}}{h} ; \\
f_{i}=\frac{m_{y} n_{i}^{(1)} \gamma_{i}+N_{i}^{(1)}}{\Delta \tau}, f_{i}^{\prime}=\frac{m_{z} n_{i}^{(2)} \gamma_{i}+N_{i}^{(2)}}{\Delta \tau} \\
\gamma_{i}=\frac{1}{M_{k}}, M_{k}=\frac{1}{\Delta \tau}+\frac{a_{1} a_{2}}{a_{2}-b N_{i}^{(k)(s-1)}}, \\
\frac{1}{M_{k}}\left[\frac{N_{i}^{(k)}}{\Delta \varepsilon}-\frac{a_{1} N_{i}^{(k)(s-1)}}{a_{2}-b N_{i}^{(k)(s-1)}}\left(1-\frac{a_{2}}{a_{2}-b N_{i}^{(k)(s-1)}}\right)\right.
\end{array}\right] \\
A_{1}=B_{1}=A_{1}^{\prime}=B_{1}^{\prime}=C_{1}=C_{1}^{\prime}=0
\end{gathered}
$$

Если пористость $m$ переменная, то она вычисляется в конечно-разностном виде по формуле $m_{i k}=m_{1}+\left(m_{0}-m_{1}\right) \xi_{i k}$.

Таким образом, определяя u, v, $\xi_{u}$, $\xi_{v}$ для одного временного слоя и подставляя их в 


\begin{tabular}{|c|c|c|c|c|c|c|}
\hline Impact Factor: & $\begin{array}{l}\text { ISRA (India) } \\
\text { ISI (Dubai, UAE } \\
\text { GIF (Australia) } \\
\text { JIF }\end{array}$ & $\begin{array}{l}=1.344 \\
=0.829 \\
=0.564 \\
=1.500\end{array}$ & $\begin{array}{l}\text { SIS (USA) } \\
\text { PИНЦ (Russia) } \\
\text { ESJI (KZ) } \\
\text { SJIF (Morocco) }\end{array}$ & $\begin{array}{l}=0.912 \\
=0.207 \\
=\mathbf{3 . 8 6 0} \\
=\mathbf{2 . 0 3 1}\end{array}$ & $\begin{array}{l}\text { ICV (Poland) } \\
\text { PIF (India) } \\
\text { IBI (India) }\end{array}$ & $\begin{array}{l}=6.630 \\
=1.940 \\
=4.260\end{array}$ \\
\hline
\end{tabular}

уравнение ионообменной сорбции находим концентрации $n^{(1)}, n^{(2)}$ для того же временного слоя.

При вычисленных искомых переменных сходимость итерационного процесса проверяется с помощью условий

$$
\begin{gathered}
\frac{\left|u_{i}^{(s)}-u_{i}^{(s-1)}\right|}{\left|u_{i}^{(s)}\right|} \leq \varepsilon, \frac{\left|v_{i}^{(s)}-v_{i}^{(s-1)}\right|}{\left|v_{i}^{(s)}\right|} \leq \varepsilon, \\
\max \left|N_{i}^{(s)}-N_{i}^{(s-1)}\right|<\varepsilon, \varepsilon>0,
\end{gathered}
$$

где $u_{i}^{(s)}, v_{i}^{(s)}, N_{i}^{(s)}, u_{i}^{(s-1)}, v_{i}^{(s-1)}, N_{i}^{(s-1)}$ - значения функции $u(x, \tau), v(x, \tau), N(x, \tau)$ в вычисляемом и предыдущем временных слоях.

\section{Обсуждение результатов}

Для проведения вычислительных экспериментов на ЭВМ и определения основных параметров процесса и их диапазонов изменения разработано программное средство. Результаты проведенных численных расчетов приведены на рис. 2-9.

Как видно из проведенных численных расчетов на ЭВМ (рис.2), скорость фильтрования суспензии по глубине фильтровальной перегородки резко уменьшается на верхних слоях фильтра, а далее она остается постоянной. Такая тенденция наблюдается при различных интервалах времени процесса фильтрования суспензии через пористую среду. Средняя скорость фильтрования суспензии за счет кольматации пор фильтра со времени убывает (кривые рис. 2). Вычислительные эксперименты также были проведены при различных значениях толщины фильтровальной перегородки и установлено, что с ростом толщины фильтра скорость заполнения порового пространства фильтровальной перегородки гель-частицами снижается, а за счет роста силы сопротивления скорость фильтрования и время работы фильтра уменьшатся.

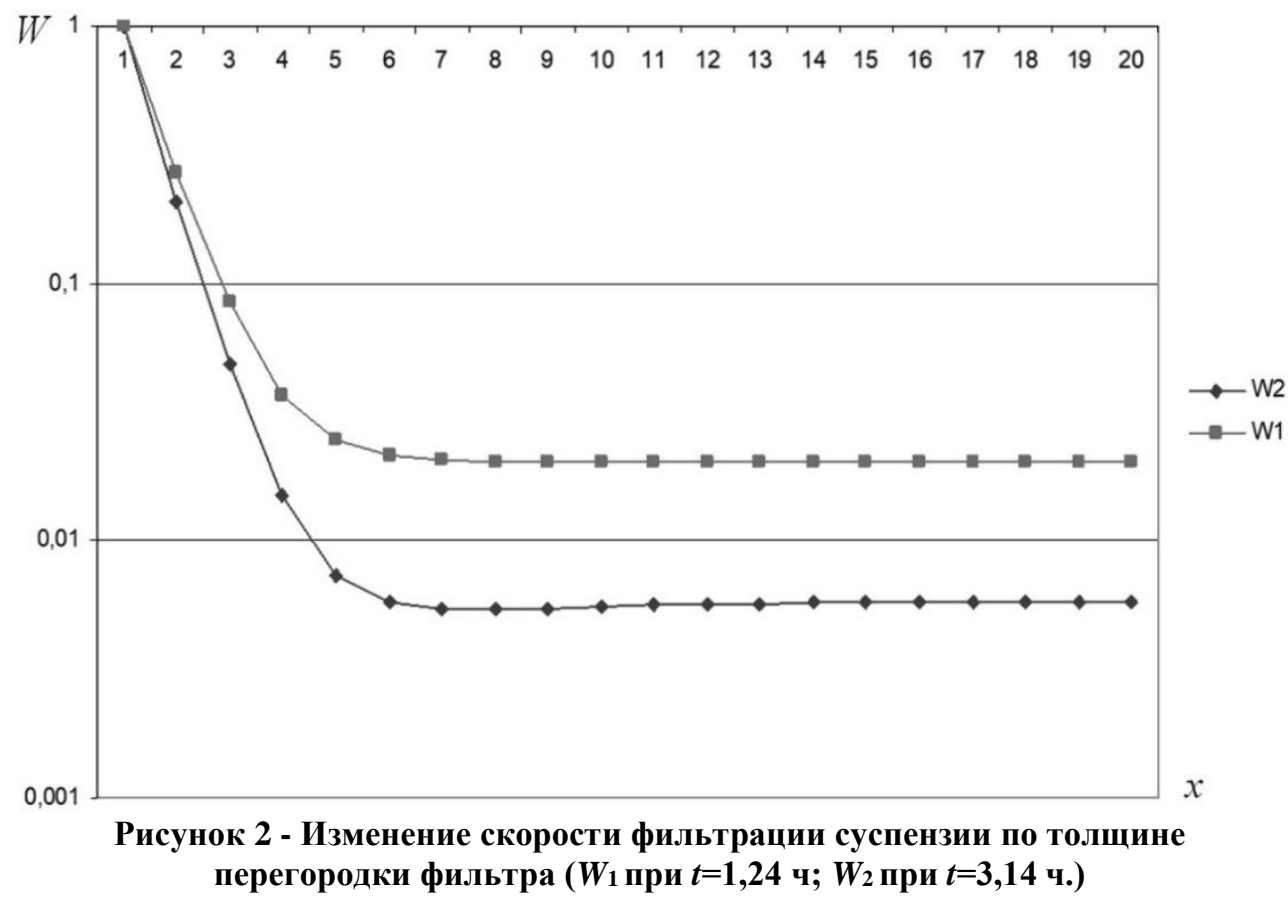

На рис. 3 приведено изменение концентрации суспензии по длине перегородки фильтра. Как следует из проведенных численных расчетов на ЭВМ концентрация суспензии на верхних слоях фильтра выше, чем на нижних слоях при начальных стадиях процесса фильтрования. С ростом время фильтрования за счет накопления и осаждения частиц внутри перегородки фильтра растет концентрация суспензии.
На рис. 4-5 приведены скорости осаждения частиц по толщине фильтровальной перегородки фильтра. Как следует из кривых рис. 4-5 максимальное осаждение частиц происходит в верхних слоях фильтра. С течением времени фильтрования за счет гидравлического роста давления внутри фильтровальной колонки происходит закупоривание пор фильтра и образование слоя осадка на его поверхности. 


\begin{tabular}{l|lrl|l|ll} 
& ISRA (India) & $=\mathbf{1 . 3 4 4}$ & SIS (USA) & $=\mathbf{0 . 9 1 2}$ & ICV (Poland) & $=\mathbf{6 . 6 3 0}$ \\
Impact Factor: & ISI (Dubai, UAE) $=\mathbf{0 . 8 2 9}$ & PUHU (Russia) $=\mathbf{0 . 2 0 7}$ & PIF (India) & $=\mathbf{1 . 9 4 0}$ \\
& GIF (Australia) & $\mathbf{0 . 5 6 4}$ & ESJI (KZ) & $=\mathbf{3 . 8 6 0}$ & IBI (India) & $\mathbf{4 . 2 6 0}$
\end{tabular}
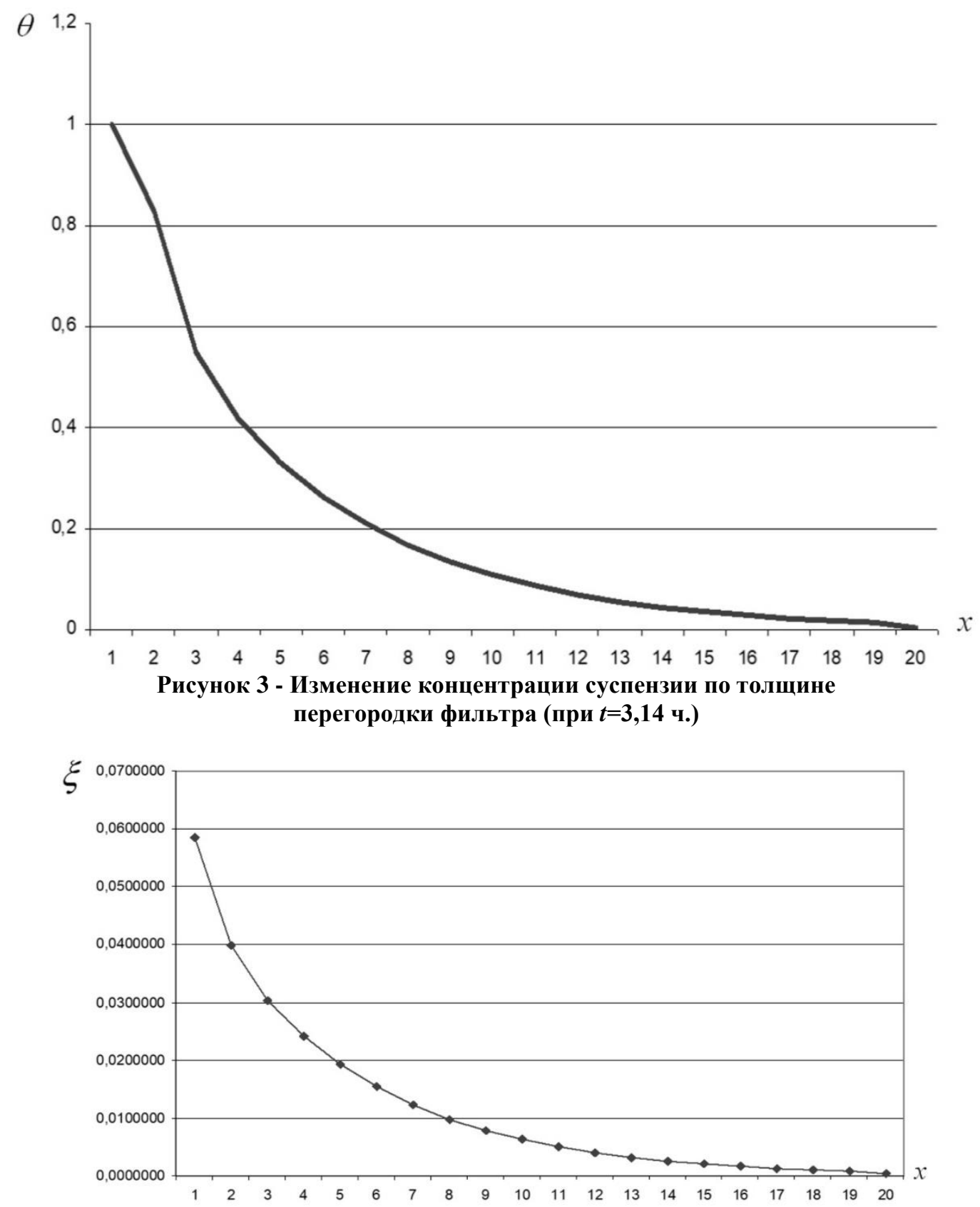

Рисунок 4 - Скорость осаждения гель-частиц внутри фильтровальной перегородки (при $t=1,24$ ч.) 


\begin{tabular}{l|lrl|l|ll} 
& ISRA (India) & $=\mathbf{1 . 3 4 4}$ & SIS (USA) & $=\mathbf{0 . 9 1 2}$ & ICV (Poland) & $=\mathbf{6 . 6 3 0}$ \\
Impact Factor: & ISI (Dubai, UAE) $=\mathbf{0 . 8 2 9}$ & PUHU (Russia) $=\mathbf{0 . 2 0 7}$ & PIF (India) & $=\mathbf{1 . 9 4 0}$ \\
& GIF (Australia) & $\mathbf{0 . 5 6 4}$ & ESJI (KZ) & $=\mathbf{3 . 8 6 0}$ & IBI (India) & $\mathbf{4 . 2 6 0}$
\end{tabular}

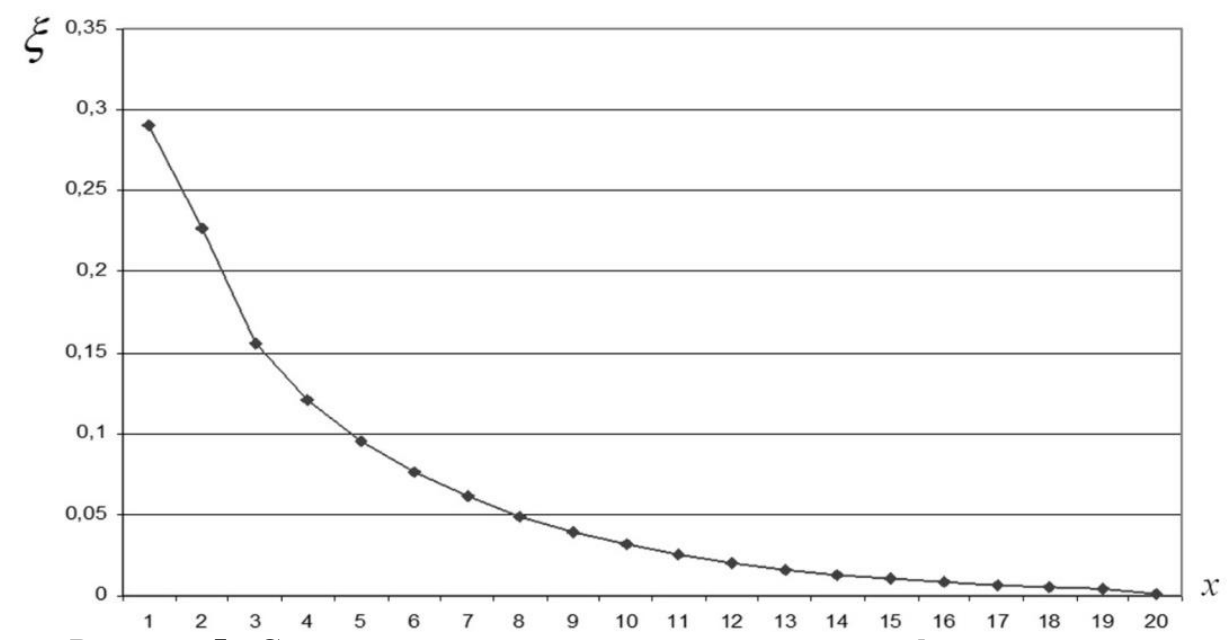

Рисунок 5 - Скорость осаждения гель-частиц внутри фильтровальной перегородки (при $t=3,14$ ч.)

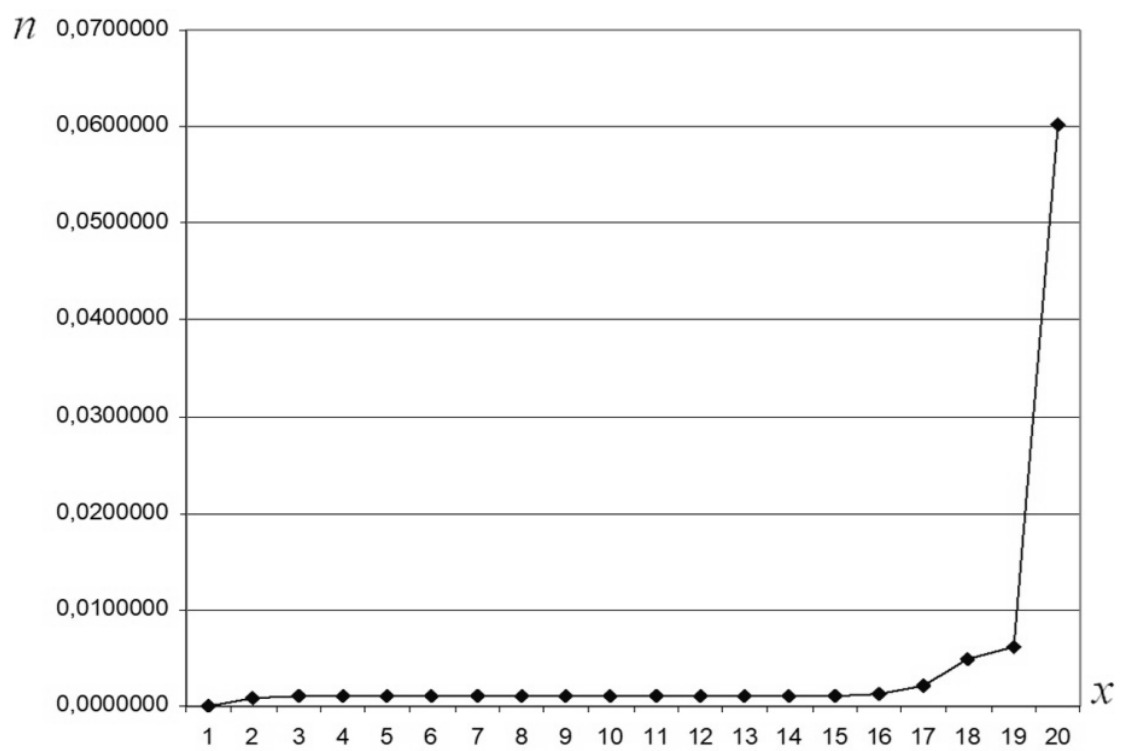

Рисунок 6 - Изменение концентрации обменивающихся ионов в растворе по длине сорбционной колонки (при $t=1,24$ ч.)

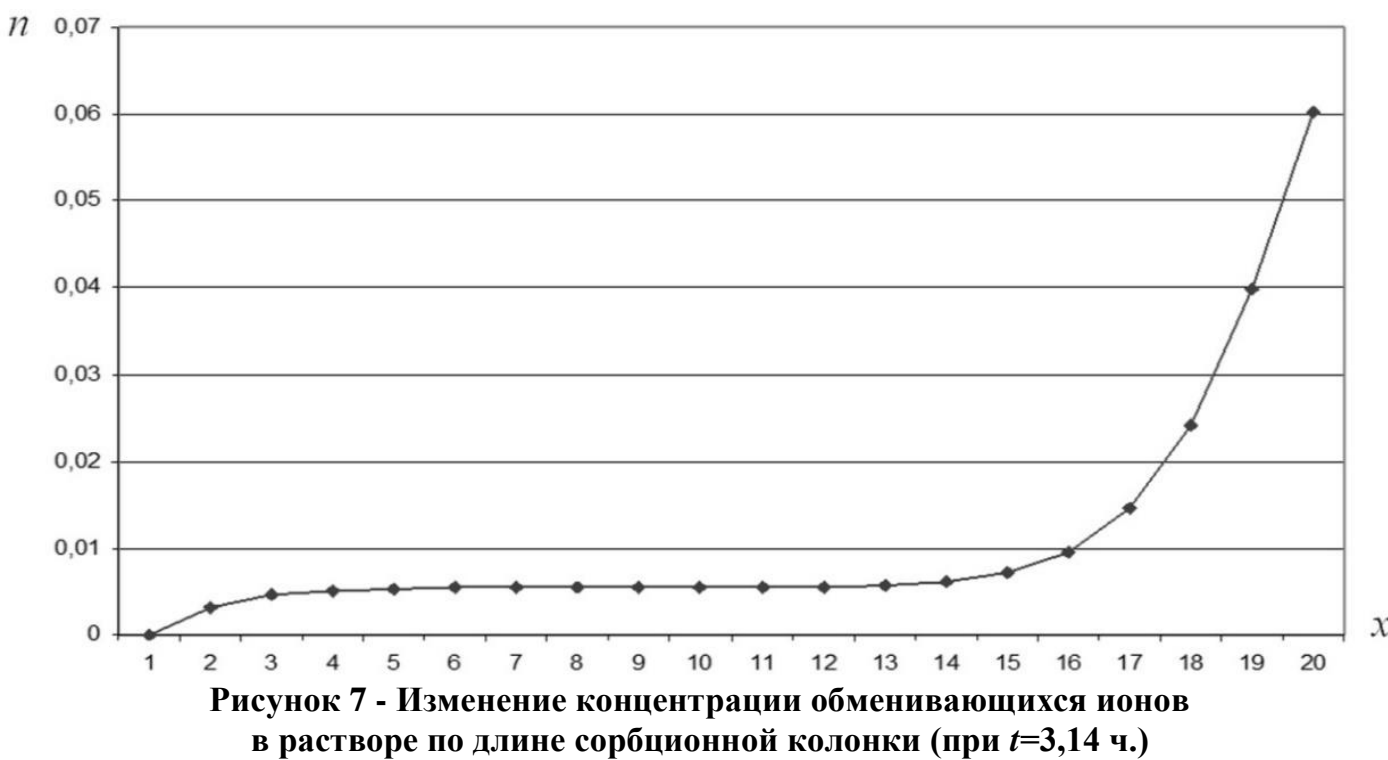

ISPC Technological development, 


\begin{tabular}{l|lrl|l|ll} 
& ISRA (India) & $=\mathbf{1 . 3 4 4}$ & SIS (USA) & $=\mathbf{0 . 9 1 2}$ & ICV (Poland) & $=\mathbf{6 . 6 3 0}$ \\
Impact Factor: & ISI (Dubai, UAE) $=\mathbf{0 . 8 2 9}$ & PUHU (Russia) $=\mathbf{0 . 2 0 7}$ & PIF (India) & $=\mathbf{1 . 9 4 0}$ \\
& GIF (Australia) & $\mathbf{0 . 5 6 4}$ & ESJI (KZ) & $=\mathbf{3 . 8 6 0}$ & IBI (India) & $\mathbf{4 . 2 6 0}$
\end{tabular}
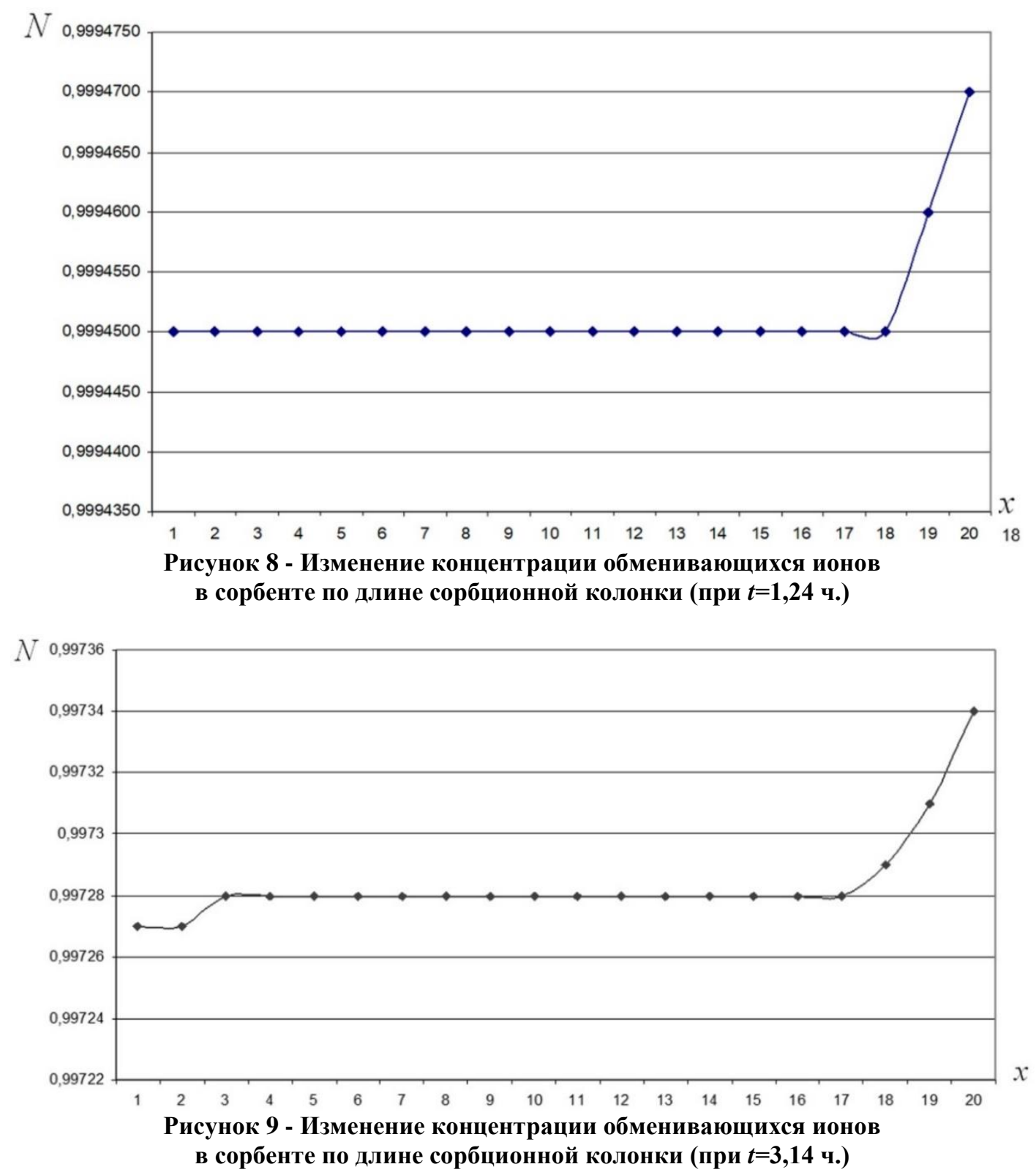

На рис. 6-7 приведены результаты проведенных численных расчетов на ЭВМ, динамика изменения концентрации обменивающихся ионов в растворе по длине сорбционной колонки фильтра при времени фильтрования $t=1,24$ ч и $t=3,14$ ч. Анализ полученных расчетов показал, что максимальное накопление концентрации обменивающихся ионов в растворе соответствует $\mathrm{x} \geq 0,75$ (рис. 7), то есть в глубине фильтровальной перегородки агрегата. С течением времени фильтрования постепенно растет концентрация обменивающихся ионов в верхних слоях фильтра.

На рис. 8-9 проиллюстрированы результаты проведенных численных расчетов на ЭВМ, рост изменения концентрации обменивающихся ионов в сорбенте по длине сорбционной колонки фильтра.

Анализ проведенных численных расчетов показал, что на начальной стадии процесса фильтрования (при $t=4-5 \quad$ ч.) выходная концентрация взвешенных гель-частиц в растворе будет незаметно уменьшаться, а при $t=5$ ч она экспоненциально убывает. Резкое уменьшение концентрации частиц в растворе связано, вопервых, с насыщением пор фильтра гель частицами, во-вторых, с ростом толщины слоя осадка на поверхности фильтра. Образовавшийся слой осадка, в свою очередь, выполняет функцию фильтра. Как следует из результатов ВЭ, время забивания пор фильтра гель-частицами зависит от скорости прохода и первоначальной концентрации фильтрата, а также от диаметров 
гель-частиц в растворе. Скорость осаждения гельчастиц в порах фильтра при $t=3$ ч будет расти по экспоненциальному закону. Полное насыщение пор фильтровальной колонки происходит при времени фильтрования $t=10,5$ ч.

Из анализа проведенных ВЭ следует, что выходная концентрация $\theta_{3}$ взвешенных частиц в фильтрате со временем убывает. Это связано, вопервых, с тем, что образовавшийся слой осадка и его переупаковка под действием поверхностной силы давления на поверхности агрегата в дальнейшем выполняет роль фильтра и, вовторых, с заполнением пор фильтра взвешенными частицами, что не дает возможности прохода гель-частиц через фильтр. При времени фильтрования $t=10$ ч значение $\square_{3}$ будет равно 0,01 .

\section{Заключение}

Разработаны математическая модель и численный алгоритм для проведения комплексного исследования процесса ионообменного фильтрования жидкостей через пористую среду, при задания различных режимов работы фильтровального агрегата и физикохимических свойств ионных растворов.

Проведенными численными расчетами установлено, что скорость фильтрования суспензии по глубине фильтровальной перегородки резко уменьшается на верхних слоях фильтра, а далее она остается постоянной по глубине фильтровальной перегородки в зависимости от толщины фильтра и диаметров гель-частиц, находящихся в суспензии.

Вычислительным экспериментом установлено, что с ростом толщины фильтра скорость заполнения порового пространства фильтровальной перегородки гель-частицами снижается, а за счет роста силы сопротивления скорость фильтрования и время работы фильтра уменьшатся, значение же $\theta_{3}$ взвешенных частиц в фильтрате со временем убывает по экспоненциальному закону.

\section{References:}

1. Burkert C., Barbosa G., Mazutti M. (2011) Mathematical modeling and experimental breakthrough curves of cephalosporin $\mathrm{C}$ adsorption in a fixed-bed column // Process Biochemistry. - 2011. - № 46. - Pp.1270-1277.

2. Fernandez X., Rosenthal I., Anlauf H., Nirschl H. (2011) Experimental and analytical modeling of the filtration mechanisms of a paper stack candle filter // Chemical Engineering Research and Design. - 2011. Vol. 89. - Issue 12. - Pr. 2776-2784.

3. Gitisa V. et al. (2010) Deep-bed filtration model with multistage deposition kinetics // Chemical Engineering Journal. - 2010. - № 163. - Rp. 78-85.

4. Lucas S., Calvo M.P., Palencia C., Alonso E., Cocero M.J. (2007) Mathematical model of supercritical $\mathrm{CO} 2$ adsorption on activated carbon applied to adsorption scale-up // The Journal of Supercritical Fluids. - 2007. - Vol. 40. - Issue 3. - Pp.331-504.

5. Santos A., Bedrikovetsky P. (2004) Size exclusion during particle suspension transport in porous media: stochastic and averaged equations // Computational and Applied Mathematics. - 2004. - Vol. 23. - № 2-3. - Rp. 259-284.

6. Zhang J. et al. (2015) Development and validation of a novel modeling framework integrating ion exchange and resin regeneration for water treatment // Water Research. - 2015. Vol. 84. - Pp. 255-265.

7. Zhihui Yu, Tao Qi, Jingkui Qu, Yingchao Guo. (2015) Application of mathematical models for ion-exchange removal of calcium ions from potassium chromate solutions by Amberlite IRC 748 resin in a continuous fixed bed column // Hydrometallurgy. - 2015. - Vol. 158. - Pp 165-171.

8. Palvanov B.Yu. (2016) Komp'yuternaya model' i vychislitel'nyy eksperiment dlya issledovaniya protsessa mnogokratnogo ionoobmennogo fil'trovaniya suspenziy // Problemy vychislitel'noy i prikladnoy matematiki Nauchnyy zhurnal. - 2016. - №.4. - p.48-63.

9. Ravshanov N., Palvanov B.Yu. (2016) Priblizhenno-analiticheskoe reshenie zadachi tekhnologicheskogo protsessa fil'trovaniya rastvorov ot nezhelatel'nykh ionov // Issledovaniya tekhnicheskikh nauk : elektronnyy nauchnyy zhurnal. - 2016. - Vyp. 1(19). - p. 25-36.

10. Ravshanov N., Palvanov B.Yu. Muxamadiyev A. (2015) Computer modelling of process of filtering of the liquid of the ionized solutions for protection of the ecosystem from of pollution sources // TUIT Bulletin. - 2015 . - № 2. - Pp. 100-105. 


\begin{tabular}{l|lrl|l|ll} 
& ISRA (India) & $=\mathbf{1 . 3 4 4}$ & SIS (USA) & $=\mathbf{0 . 9 1 2}$ & ICV (Poland) & $=\mathbf{6 . 6 3 0}$ \\
Impact Factor: & ISI (Dubai, UAE) $=\mathbf{0 . 8 2 9}$ & PUHL (Russia) $=\mathbf{0 . 2 0 7}$ & PIF (India) & $=\mathbf{1 . 9 4 0}$ \\
& GIF (Australia) & $\mathbf{0 . 5 6 4}$ & ESJI (KZ) & $=3.860$ & IBI (India) & $=\mathbf{4 . 2 6 0}$ \\
& JIF & $\mathbf{1 . 5 0 0}$ & SJIF (Morocco) & $=\mathbf{2 . 0 3 1}$ & & \\
\hline
\end{tabular}

11. Ravshanov N., Palvanov B.Yu. (2015) Matematicheskaya model' protsessa ionoobmennogo fil'trovaniya ionizirovannykh rastvorov // Problemy informatiki i energetiki. 2015. - № 6. - p. 10-17.

12. Ravshanov N., Palvanov B.Yu. (2015) Vychislitel'nyy eksperiment dlya issledovaniya tekhnologicheskogo protsessa fil'trovaniya zhidkikh i ionizirovannykh rastvorov // Otraslevye aspekty tekhnicheskikh nauk : elektronnyy nauchnyy zhurnal. - 2015. - №3 (45). - p. 18-28.

13. Ravshanov N., Palvanov B.Y., Elmurodova B. (2016) Computer modelling of problems filtering low-concentration suspensions //
Theoretical \& Applied Science. - 2016. - № 9(41). - p. 101-110.

14. Ravshanov N., Palvanov B.Y. (2017) Numerical solution of inverse problems filtering process of low-concentration solutions // Theoretical \& Applied science. - 2017. - № 4(48). - p. 137-144.

15. Samarskiy A.A. (1971) Vvedenie v teoriyu raznostnykh skhem. -M.: Nauka, 1971. - 552 p.

16. Tikhonov A.N., Samarskiy A.A. (1977) Uravneniya matematicheskoy fiziki. - izd. 5-e, stereotip. - 1977. - 46 p.l. Available: http://samarskii.ru/books/book1971.djvu (Accessed: 10.11.2017). 